\title{
ARE ANOREXIC TENDENCIES PREVALENT IN THE HABITUAL RUNNER?
}

\author{
G. D. WHEELER, MSc* ${ }^{*}$ S. R. WALL, MA**, A. N. BELCASTRO, PhD*, P. CONGER, MA* \\ and D. C. CUMMING, MBChB, MRCOG, FRCS(C)**†
}

Depts. of *Physical Education and Sports Studies, **Obstetrics and Gynaecology and TMedicine (Division of Endocrinology), University of Alberta, Edmonton, Alberta, Canada

\begin{abstract}
To investigate whether runners displayed any of the abnormalities characteristic of patients with anorexia nervosa, we conducted a cross sectional study of 31 high mileage, 18 low mileage runners and 18 non-running controls. Subjects completed a personal data questionnaire, the Jackson Personality Inventory (JPI) and the Eating Attitudes Questionnaire (EAT), underwent a body image test and a blood sample was obtained for measurement of reproductive, thyroid and adrenal hormones.

High mileage runners scored significantly higher infrequency scores on the JPI than sedentary controls but there was no evidence of psychopathology. The high mileage runners also significantly overestimated waist width and there were small but statistically significant differences in EAT scores between controls and the runner groups. Ten of 49 runners had EAT scores beyond two standard deviations above the mean of non-running controls. Serum total, free and non-specifically bound testosterone and prolactin levels were significantly lower in high mileage runners than controls. LH, FSH, cortisol and thyroid hormones were not significantly different. There were no significant differences in any hormone between low mileage runners and controls.
\end{abstract}

The results suggested that running may have a chronic effect on serum testosterone and prolactin levels in high mileage but not low mileage runners. Although there was no significant evidence of anorexia nervosa on testing the runners with EAT, the overestimation of waist size provided some evidence of a distortion of body image in the high mileage runners. Runners displayed no clear abnormalities characteristic of patients with anorexia nervosa.

Key words: Men, Runners, Anorexia, Personality, Body image, Testosterone, Cortisol, Luteinising hormone, Follicle stimulating hormone, Prolactin, Thyroid.

\section{INTRODUCTION}

Many benefits have been associated with running. Psychological advantages include better stress management, reduction of depression, improved selfesteem, and an improved feeling of well being and a heightened sense of personal control (Lion, 1978; Greist et al, 1978; Jorgenson and Jorgenson, 1979; Leonardson, 1977). Physiological advantages include improvement in aerobic capacity, and reductions in per cent of body fat (Saltin et al, 1968; Katch et al, 1973; Pollock, 1973; Pollock et al, 1975; Wilmore et al, 1970).

Recently, however, adverse effects of distance running have been described. Overuse injuries appear to be increasing as recreational and competitive runners attempt to run further and faster (Stanish, 1984). Chronic effects of endurance running programmes on reproductive function in women have been observed (Cumming and Rebar, 1983). The subtle, less visible effects of running may potentially be more serious, and although running has been described as a "positive addiction" (Glasser, 1976). Morgan (1979) referred to the psychological dependence on running and the need to continue running against medical advice as "negative addiction".

Address for correspondence:

Dr. D. C. Cumming

5-121, Clinical Sciences Building

University of Alberta

Edmonton

Alberta

Canada T6G 2G3
Habitual or "obligatory" running in men has been compared with the anorexia syndrome in women (Sours, 1981; Yates et al, 1983). The hyperactive state and dietary practices of runners may resemble those of adolescents with anorexia nervosa. The social backgrounds of habitual runners tend to be similar to those of the typical anorexic patient and the groups may share similar personality traits and behaviours (Sours, 1981). Both the runner and the anorexic patient are said to be caught up in a partially successful, albeit dangerous, attempt to establish a self identity (Sours, 1981).

Patients with anorexia nervosa have characteristic abnormalities on psychological testing, in eating attitudes, in body image and in reproductive, thyroid and adrenal hormonal values (Schwabe, 1981). We therefore conducted a cross sectional study of 49 runners and 18 sedentary controls to see if, first, there were any significant differences between runners and sedentary controls and, second, runners had any characteristics similar to those described in patients with anorexia.

\section{SUBJECTS AND METHODS}

A total of 67 subjects volunteered for the study in response to advertisements and informed consent was obtained from each subject. The runners came from the University of Alberta and local running clubs. Runners ranged in age from 18-56 years (mean 33.6) and were divided into two groups depending upon their weekly training load. Those training $\mathbf{4 0}$ or more miles per week were described as high mileage runners (HMR) and those running 20-39 miles per week were called low mileage runners (LMR). Eighteen 
sedentary men, mean age 27.8 years (range 18-46) were accepted as controls on the basis of their having no involvement in physical exercise, organised or otherwise.

Height and weight were recorded and a ponderal index (PI) was calculated for each subject (PI = height in $\mathrm{cm} /$ cube root of weight in $\mathrm{kg}$ ). All subjects were asked to complete a questionnaire on their social background and in the case of runners only, on their attitudes to running. The questionnaire examined eating attitudes in relation to running, significance of running to daily life and responsibility to family occupation. All subjects completed the Eating Attitudes Test (EAT) (Garner and Garfinkel, 1979), the Jackson Personality Inventory (Jackson, 1976) which includes scales of social adroitness, anxiety, self-esteem, energy level, anxiety and an infrequency scale designed to check for implausible or inappropriate responses. The body image test described by Askevold (1975) was also performed. Each subject was asked to mark shoulder, waist and hip width on a large, vertically mounted blank sheet of paper in response to the investigator touching the area with both hands simultaneously. A body image estimation index was calculated for each measure using the formula PERCEIVED SIZE/REAL SIZE $\dot{x} 100$. A composite body image estimation index was also calculated in a similar manner. Reliability of the body image test was assessed by the correlation of two tests, one carried out prior to the testing session and the other at its conclusion.

A single, non-fasting blood sample was obtained from each subject by venepuncture between 16.00 and 18.00 hours following a light caffeine-free lunch. The runners had not exercised in the previous 24 hours. The blood sample was allowed to clot at room temperature and the serum was separated by centrifugation and stored at $-20^{\circ} \mathrm{C}$ until assayed. Luteinising hormone (LH), follicle stimulating hormone (FSH), prolactin, cortisol, total testosterone, thyroid stimulating hormone (TSH), total thyroxine $\left(T_{4}\right)$ triiodothyronine $\left(\mathrm{T}_{3} \mathrm{U}\right)$ and free thyroxine index (FTI) were measured using commercially available radioimmunoassay kits. Free testosterone, non sex-hormone bound testosterone and sex-hormone binding globulin were measured as described previously (Wheeler et al, 1984). All samples were measured in a single assay for each hormone. Intra-assay variability was in all cases less than $10 \%$.

Data were analysed using a standard analysis of variance programme comparing controls with runners (Sokal and Rohlf, 1981). When analysis of variance indicated significant differences, comparisons between the individual group means in the runners groups with the means in the control group means and was carried out using the Dunn-Sidak method (Sokal and Rohlf, 1981). A Pearson product moment correlation was calculated for replicate body image measures.

\section{RESULTS}

There was no significant difference in mean height and weight between runners and controls (Table I). Measures of PI were similar as the runners tended to be both shorter and lighter than the controls. None of the measures to be described correlated with age, height, weight or ponderal index.

In response to the running questionnaire, twenty-four
TABLE I

Height, woight and ponderal index (PI) (mean \pm SEM) in sedentary controls, low mileage runners (LMR) and high mileage runners (HMR)

\begin{tabular}{lcccc}
\hline Group & $n$ & Height (cm) & Weight (kg) & PI \\
\hline Controls & 18 & $183.2 \pm 2.8$ & $76.2 \pm 6.1$ & $12.9 \pm 0.1$ \\
LMR & 18 & $176.5 \pm 1.7$ & $67.4 \pm 2.0$ & $12.7 \pm 0.1$ \\
HMR & 31 & $177.4 \pm 0.9$ & $67.9 \pm 1.0$ & $13.0 \pm 0.2$ \\
\hline
\end{tabular}

per cent of runners reported running more than once per day and $18 \%$ reported that they ran more than 10 times per week. Fifty-two per cent competed frequently in races, $13 \%$ at every opportunity. In contrast, only $4 \%$ never competed at all. Eighteen per cent reported regularly missing a meal to run with $55 \%$ occasionally missing a meal. Sixty-three per cent reported regularly eating 3 meals per day and $74 \%$ reported that they never felt the need to run directly after a meal. Half of the runners reported that running was a significant and important part of their lifestyle with $20 \%$ indicating that they would ignore medical advice to stop running. Thirty per cent said that they would experience an extreme sense of loss if they had to stop running although only $4 \%$ indicated that weight gain would be a primary concern. Thirteen per cent of the runners said that running often caused them to be late for work or to neglect family responsibilities. The demographic questionnaire revealed that $96 \%$ of the runners were in social class I with $85 \%$ having a bachelor degree or higher. Eighty-six per cent reported that they came from high achievement orientated families.

Two personality scales in the JPI revealed significant differences among the groups. Compared with controls, HMR scored significantly higher energy level scores (52.2 \pm 2.63 vs $45.7 \pm 2.28, p<0.05)$ and significantly higher infrequency scores $(54.1 \pm 1.5$ vs $47.0 \pm 1.0, p<0.05)$. The infrequency scores of LMR were not significantly different from those of the control group.

Runners had significantly higher EAT scores on anova ( $p<0.05$ ), although no differences between controls and individual runner groups were observed. HMR scored $9.6 \pm$ 0.8 , LMR $10.4 \pm 1.3$ and controls $7.4 \pm 1.2$. No group mean approached the score of 30 which has been described as indicative of anorexia nervosa (Garner and Garfinkel, 1979). Three runners scored the moderately high values of 21,23 and 24 points on the EAT. There was considerable polarisation of the results in the runners. Eight runners scored less than five points, while ten runners exceeded two standard deviations above the mean of normal controls. In contrast, only one control scored less than five and none had scores higher than two standard deviations above the mean.

HMR overestimated their waist width compared with values in control subjects (Table II, $\mathrm{p}<0.05$ ). Othe measures of body image were not significantly different. There were no significant differences in any body image test between LMR and control groups. Correlations between the first and second measures of body image parameters were positive and significant for all groups and the statistical analysis was consistent for individual measures comparing the two tests.

We have previously reported that total, free and non- 


\section{TABLE \|}

Body image testing (mean \pm SEM): values are calculated as an index of PERCEIVED SIZE/REAL SIZE $\times 100$. Reeults are shown for controls, low mileage runners (LMR) and high mileage runners (HMR)

\begin{tabular}{lcccc}
\hline Group & Composite & Shoulder & Waist & Hip \\
\hline Controls & $102.9 \pm 4.3$ & $92.6 \pm 3.8$ & $105.4 \pm 5.2^{*}$ & $111.1 \pm 5.5$ \\
LMR & $109.8 \pm 4.2$ & $97.3 \pm 4.2$ & $113.6 \pm 5.2$ & $118.5 \pm 4.6$ \\
HMR & $115.9 \pm 4.5$ & $99.3 \pm 3.4$ & $127.3 \pm 6.2^{*}$ & $120.2 \pm 5.8$ \\
\hline
\end{tabular}

* $p<\mathbf{0 . 0 5}$ on comparison of means

SHBG bound testosterone (Fig. 1) and prolactin (Fig. 2) levels were significantly lower in HMR compared with the control group (Wheeler et al, 1984). In contrast, although testosterone and prolactin values in LMR were reduced, the difference failed to reach statistical significance (Figs. 1 and 2). There were no other significant differences among the groups in other hormones (Table III).

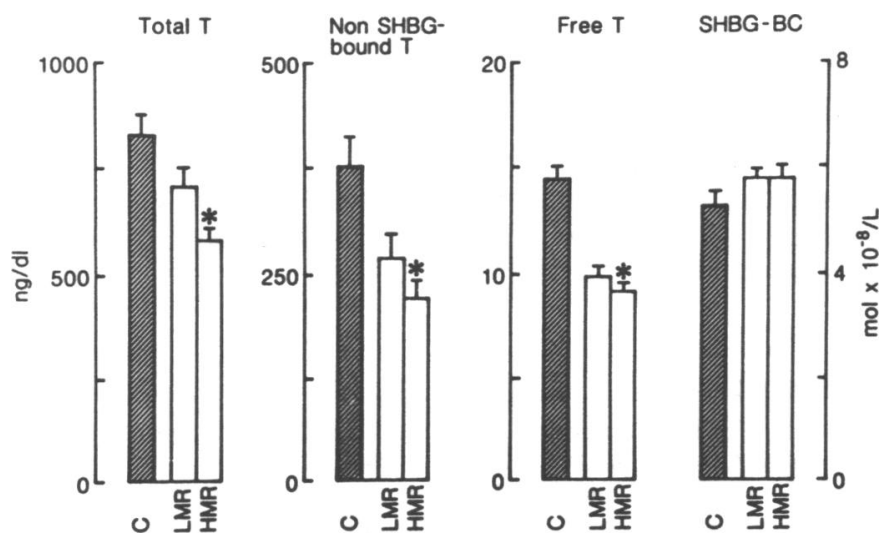

Fig. 1: Mean ( \pm SEM) serum levels of total testosterone, non sex-hormone binding globulin bound testosterone (non-SHBG), free testosterone and sex hormone binding globulin (SHBG) in sedentary controls (shaded bar) and in low and high mileage runners (white bars, respectively LMR and HMR). Asterisk indicates significantly different from controls $(p<0.05)$.

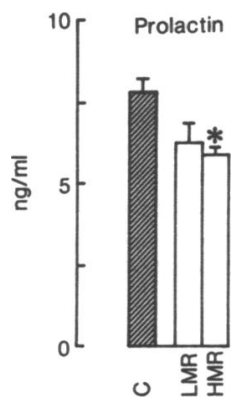

Fig. 2: Mean (士 SEM) serum levels of prolactin in sedentary controls (shaded bar) and in low and high mileage runners (white bars, respectively LMR and HMR). The asterisk indicates significantly different from control values $(p<0.05)$.

\section{DISCUSSION}

Recent increases in the frequency of diagnosis of anorexia nervosa (Yates et al, 1983; Garner et al, 1983; Jones et al, 1980; Kalucy et al, 1977; Shainess, 1979) and the rapid growth of the fitness movement have been accompanied by reports of the behavioural similarities in anorexics and habitual exercise enthusiasts (Sours, 1981; Yates et al, 1983; Henry, 1982). Early comparisons centred on high school athletes (Smith, 1980) and women runners (Henry, 1982). More recently, Yates and colleagues (1983) described in an anecdotal fashion, the demographic, behavioural and psychological similarities in a group of male obligatory runners and women with anorexia nervosa. The symptoms of anorexia are similar in both sexes although there is a female to male ratio of 10 or 20 to one (Bruch, 1971; Crisp and Toms, 1972; Hay and Leonard, 1979). The onset of anorexia is peripubertal while most obligatory men runners are in their third or fourth decade when they begin running. The importance of developing sexuality may be the trigger for anorexia at adolescence, while the runners are possibly motivated by the fear of waning effectiveness (Yates et al, 1983). The terms "running anorexia" and "anorexia athletica" have been used recently to describe this association.

Hyperactivity in the anorexic patient has frequently been cited as an interesting occurrence but not a diagnostic criterion (Blitzer et al, 1961; Boyar, 1978; Epeling et al, 1983; Hogan et al, 1974). Feighner and colleagues (1972) described activity associated with anorexia as one of six secondary symptoms. The levels of activity in runners in the present study were high with several running ten to fourteen times per week. Not surprisingly, the runners in both groups had higher energy level scores on the JPI scale than non-exercising controls. High levels of physical activity are used in the anorexic patient to accelerate weight loss. Rats with free access to an exercise wheel have been reported to become anorexic and highly active when diatary restriction was imposed (Epeling et al, 1981, 1983; Routtenberg and Kusnezof, 1967). Self-imposed dietary restrictions and an increased exercise load could possibly produce an analogous situation in some runners resulting in anorexic tendencies (Epeling et al, 1983). There were no indications that the subjects in the present study began running in order to lose weight nor that they were presently concerned with body weight or food intake. Only two runners expressed concern over possible weight gain on stopping their running. In contrast, a substantial number of runners $(18 \%)$ were prepared to skip meals regularly in order to run.

Although there were small but statistically significant differences between scores of runners and controls, the EAT provided no objective evidence that the runners, individually or as groups, had anorexic tendencies, a finding which is in contrast to a study of women college

TABLE

Serum levels (mean \pm SEM) of luteinising hormone (LH), follicle stimulating hormone (FSH), thyroid stimulating hormone (TSH), thyroxine (T, ), triliodothyronine uptake $\left(T_{3} U\right)$ and free thyroxine (FT,

\begin{tabular}{lccccccc}
\hline Group & LH,mlU/ml & FSH,mlU/ml & Cortisol,ug\% & TSH,mlUn & $T_{4}, \mathrm{nmol} /$ & $T_{3} \mathrm{U}$ & FT, \\
\hline Controls & $9.6 \pm 1.0$ & $5.1 \pm 0.7$ & $15.9 \pm 1.8$ & $4.8 \pm 0.2$ & $114.7 \pm 6.7$ & $39.7 \pm 1.0$ & $95.2 \pm 4.3$ \\
LMR & $6.7 \pm 0.7$ & $9.3 \pm 2.4$ & $13.8 \pm 1.7$ & $5.9 \pm 0.6$ & $105.7 \pm 6.3$ & $41.8 \pm 1.3$ & $91.9 \pm 4.3$ \\
HMR & $9.1 \pm 0.8$ & $6.6 \pm 0.9$ & $12.9 \pm 1.1$ & $4.9 \pm 0.2$ & $106.7 \pm 5.1$ & $41.8 \pm 0.8$ & $92.3 \pm 3.4$
\end{tabular}


runners (Henry, 1982). Anecdotal evidence of abnormal dietary behaviour and concern over caloric intake has been provided by Sours (1981) and Yates and colleagues (1983). Individual runners in the present study approached the anorexic score but, surprisingly, several runners had very low scores on the EAT. No direct measurement of caloric intake was undertaken, therefore we could not evaluate the accuracy of the information provided by the runners on the EAT scores. There was some discrepancy between questionnaire information and anecdotal information gathered during the study. The high score gained on the JPI Infrequency scale together with the improbably low scores on the EAT in some runners raises the question of whether there was some degree of denial. Although patients with anorexia nervosa deny their emaciation they do not generally deny their dietary practices. It may be that the EAT is an inappropriate means of evaluating dietary practices in male runners. Perhaps a more relevant area of concern for denial would be the need to continue running against medical advice. While the observation that $20 \%$ of runners would do so supports the concept of denial, the answer to a "what if" question is hardly conclusive.

The distortion of body image in anorexic patients is well documented (Garner et al, 1976; Gasper et al, 1979; Pierloot and Houben, 1978; Slade and Russell, 1973). Normal individuals over-estimate body dimensions to some degree (Pierloot and Houben, 1978). The significant overestimation of waist width by HMR may reflect a focus of concern on waist size in the runners, since previous evidence has suggested that individuals overestimate that part of the body which is of most concern to them (Askevold, 1975).

Psychological testing has shown that anorexic patients, although not having a distinct personality profile, tend to have low self-esteem, high anxiety, low social adroitness and excessive shyness (Halmi, 1974; Kay and Leigh, 1954). From the responses to the JPI, there were no similarities between the runners and the anorexic profile. Our findings are, therefore, in agreement with those of Blumenthal and colleagues (1984) who found clear differences between runners and anorexics on the Minnesota MultiPhasic Personality Inventory. We found no evidence to suggest that a high percentage of runners regularly neglected their occupation and family responsibilities, a finding in contrast to the anecdotal evidence provided by Morgan (1979) and Sours (1981). The social background of anorexic patients has been described as predominantly social class I and II associated with high achievement and achievement motivation (Garner et al, 1983; Jones et al, 1980; Halmi, 1974; Crisp et al, 1976). The present and previous studies have provided consistent evidence that runners come from the same background (1983). It may be argued that those engaged in manual work may be less inclined to seek a less strenuous physical activity so that the runners may be a self-selecting population.

The endocrine profile of the anorexic patient shows marked abnormalities (Boyar, 1978; Brown et al, 1977; Vigersky, 1977; Warren and Vandeviele, 1973). The endocrine profile of runners in the present study did not resemble that described in anorexic males although there were some interesting differences between the HMR and control groups. The mechanism for such a change remains unclear and the concentrations of steroid hormones may be altered by such diverse factors as physical and psychological stress, diet and body composition, all of which may be operative in runners.

In conclusion, the results did suggest subtle alterations in the pituitary-gonadal axis and in the body image of high mileage runners. Despite these findings and although ten of 49 runners had an EAT score which was above two standard deviation above the mean of sedentary controls, there was no evidence that any runner met the criteria for diagnosis of anorexia nervosa. There is little doubt that many runners are committed to the sport of running but there seems to be little evidence that the dedicated runner can be compared with the anorexic patient obsessed with the pursuit of thinness. Whilst physical activity may have a mild anorexigenic effect it seems more likely that genetic and environmental characteristics are necessary predisposing factors to the development of the true anorexia nervosa syndrome. it also seems possible that the many anecdotal accounts of athletes with anorexia nervosa perhaps could be represented as patients with anorexia who express their hyperactivity in a socially acceptable manner. The answers to these questions remain to be elucidated.

\section{ACKNOWLEDGEMENTS}

This study was supported by grants from the Central Research Fund of the University of Alberta and the Special Services and Research Fund of the University of Alberta Hospital.

\section{References}

Askevold, F., 1975 "Measuring body image. Preliminary report on a new method". Psychother.Psychosom. 26: 71-77.

Blitzer, J. R., Rollins, N. and Blackwell, A., 1961 “Children who starve themselves". Psychosom. Med. 23: 369-383.

Blumenthal, J. A., O'Toole, L. C. and Chang, J. L., 1984 "Is running an analogue of anorexia nervosa". JAMA 1984; 252: 520-523.

Boyar, R. M., 1978 "Endocrine changes in anorexia nervosa".

Med.Clin.N.Amer. 62: 227-303.

Brown, G. M., Garfinkel, P. E., Jeunecwiz, N., Moldofsky, H. and Stancer, H. C. 1977 "Endocrine profiles in anorexia nervosa" In: Vigersky, R., ed. Anorexia Nervosa. New York: Raven Press, 123-135.

Bruch, H., 1971 "Anorexia nervosa in the male". Psychosom.Med. 33: 31-47.

Casper, R. C., Halmi, K., Goldberg, S., Eckert, E. and Davis, J., 1979 "Disturbance of body image estimation as related to other characteristics and outcome in anorexia nervosa". Brit.J.Psychiatr. 134: 60-66.

Crisp, A. H. and Toms, D. A., 1972 "Primary anorexia nervosa or weight phobia in the male". Brit.Med.J. 1: 334-338.

Crisp, A. H., Palmer, R. L. and Kalucy, R. S., 1976 "How common is anorexia nervosa". Brit.J.Psychiat. 128: 549-554.

Cumming, D. C. and Rebar, R. W., 1983 "Exercise and reproductive function in women". Am.J.Indust.Med. 4: 113-125.

Epeling, W. F., Pierce, W. D. and Stefan, L., 1981 "Schedule induced self starvation: possible implications for anorexia nervosa" In: Bradshaw, C. M., Szabadi, E. and Lowe, C. F., eds. Quantification of Steady-State Operant Behaviour. Amsterdam: Elsevier-N. Holland Biomedical, 393-397.

Epeling, W. F., Pierce, D. W. and Stefan, L., 1983 "A theory of activity-based anorexia". Int.J.Eat Dis. 3: 27-46.

Feighner, J. P., Robins, E., Guze, S. B., Woodruff, R. A., Winokur, G. and Munoz, R., 1972 "Diagnostic criteria for use in psychiatric research". Arch.Gen.Psychiatr. 26: 57-63.

Garner, D. M., Garfinkel, P. E., Stance, H. L. and Moldofsky, H., 1976 "Body image disturbances in anorexia nervosa and obesity". Psychosom. Med. 38: 327-356.

Garner, D. M. and Garfinkel, P. E., 1979 "The Eating Attitudes Test: an index of symptoms of anorexia nervosa". Psych.Med. 9: 273-279.

Garner, D. M., Garfinkel, P. E. and Olmsted, P., 1983 "An overview of sociocultural factors in the development of anorexia nervosa". In: Darby, P., Garfinkel, P., Garner, D. M. and Coscina, D., eds. Anorexia Nervosa: Recent Developments in research". New York, NY: Alan Liss Inc. 65-82.

Glasser, W. 1976 "Positive addiction". New York: Harper and Row.

Greist, J. H., Klein, M. H., Eischens, R. R. and Faris, J. T., 1978 "Running out of depression". Phys.Sportsmed 6 (12): 49-51. 
Halmi, K., 1974 "Anorexia nervosa. Demographic and clinical features". Psychosom. Med. 36: 18-26.

Hay, G. G., Leonard, J. C., 1979 "Anorexia nervosa in males". Lancet 2: 574-576.

Henry, S. J. P., 1982 "The price of perfection". The Runner 4 (6): 35-39.

Hogan, W. M., Huerta, E. and Lucas, A. R., 1974 "Diagnosing anorexia nervosa in males". Psychosomatics 15: 122-126.

Jackson, D. N., 1976 "Jackson Personality Inventory Manual". Goshen, NY: Res.Psychol. 1-40.

Jones, D. J., Fox, M., Babigian, M. and Hulton, H. E., 1980 "Epidemiology of anorexia in Monroe County, NY. 1960-1976". Psychosom.Med. 42: 551-558.

Jorgenson, C. B. and Jorgenson, D. E., 1979 "The effort of running on perception of self and others". Percept. Motor Skills 48: 242.

Kalucy, R. C., Crisp, A. H., Lacy, J. H. and Harding, B., 1977 "Prevalence and prognosis in anorexia nervosa". Aus.NZ.J.Psych. 11: 251-257.

Katch, F. W., Philips, W., Carter, J. E. L., Boyer, J. L., 1973 “Cardiovascular changes in middle aged men in two hours of training". J.Appl.Physiol. 34: 57-59.

Kay, D. W. K. and Leigh, D., 1954 "The natural history, treatment and prognosis of anorexia nervosa". J.Ment.Sci. 100: $411-431$.

Leonardson, G. R., 1977 "Relationship between self concept and perceived physical fitness". Percept. Motor Skills 44:62.

Lion, L. S., 1978 "Psychological effects of jogging". Percept.Motor Skills 47: $1215-1218$.

Morgan, W. P., 1979 "Negative addiction in runners". Phys.Sports Med. 7 (2): 56-70.

Pierloot, R. A. and Houben, M. E., 1978 "Estimation of body dimensions in anorexia nervosa". Psycholog. Med. 8: 317-324.

Pollock, M. L., 1973 "The quantification of endurance training programmes". Exer.Spts Sci.Rev. 1: 155.

Pollock, M., Dimmick, J., Miller, H., Zendrick, Z. and Linnerud, A., 1975 "Effects of mode of training on cardiovascular function and body composition of adult men". Med.Sci.Sports Exer. 7: 134-145.
Routtenberg, A. and Kuznezof, A. W., 1976 "Self-starvation of rats living in activity wheels on a restricted feeding schedule". J.Comp.Physiol.Psychol. 64: 414-421.

Saltin, B., Blomquist, B., Mitchell, J. H., Johnson, R. L. Jr., Wildenthal, K. and Chapman, C. B., 1968 "Response to submaximal and maximal exercise after bed rest and training". Circulation 38 (suppl. 7): 1-78.

Schwabe, A. D., Lippe, B. M., Chang, R. J., Pops, M. A. and Yager, J., 1981 "Anorexia nervosa". Ann.Int.Med. 94: 371-381.

Shainess, N., 1979 "The swing of pendulum from anorexia to obesity". Amer.J.Psychoanal. 39: 225-234.

Slade, P. D. and Russell, G. F. M., 1973 "Awareness of body dimensions in anorexia nervosa. Cross sectional and longitudinal studies". Psychol.Med. 3: 188-199.

Smith, N. D., 1980 "Excessive weight loss and food aversion in athletes simulating anorexia nervosa". Pediatr. 66: 139-142.

Sokal, R. R. and Rohlf, F. J., 1981 "Single classification analysis of variance. Comparison among means" In: Biometry. San Francisco, Ca.: W. H. Freeman and Co., 232-242.

Sours, J. A., 1981 "Running: anorexia nervosa and perfection" In: Sachs M. H. and Sachs, M. H., eds. Psychology of Running. Champaign, III.: Human Kinetic Publishers, 80-91.

Stanish, W., 1984 "Overuse injuries in athletes: a perspective". Med.Sci.Sports Exer. 16: 1-7.

Vigersky, R., 1977 In: Vigersky, R., ed. Anorexia Nervosa. New York: Raven Press, 149-161.

Warren, M., Vandewiele, R. C., 1973 "Clinical and metabolic features of anorexia nervosa". Am.J.Obstet.Gynecol. 117: 435-449.

Wheeler, G. D., Wall, S. R., Belcastro, A. N. and Cumming, D. C., 1984 "Reduced serum testosterone and prolactin levels in male distance runners". JAMA 252: 514-516.

Wilmore, J., Royce, J., Girandola, R., Katch, F. and Katch, V., 1970 "Body composition changes with a 10 week programme of jogging". Med.Sci.Sports Exer. 2: 113-117.

Yates, A., Leehay, K. and Shisslak, C. M., 1983 "Running: an analogue of anorexia nervosa". N.Eng.J.Med. 308: 251-255. 